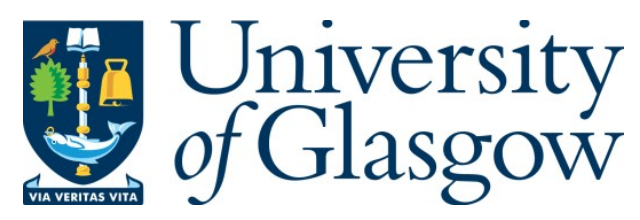

Béal, S., Deschamps, M. and Moulin, H. (2020) Taxing congestion of the space commons. Acta Astronautica, 177, pp. 313-319.

(doi: 10.1016/j.actaastro.2020.07.036)

This is the Author Accepted Manuscript.

There may be differences between this version and the published version. You are advised to consult the publisher's version if you wish to cite from it.

https://eprints.gla.ac.uk/221526/

Deposited on: 1 August 2020

Enlighten - Research publications by members of the University of Glasgow http://eprints.gla.ac.uk 


\title{
Taxing Congestion of the Space Commons ${ }^{\text {th }}$
}

\author{
Sylvain Béal ${ }^{\mathrm{a}}$, Marc Deschamps ${ }^{\mathrm{a}}$, Hervé Moulin ${ }^{\mathrm{b}, *}$ \\ ${ }^{a}$ CRESE EA3190, Univ. Bourgogne Franche-Comté, F-25000 Besançon, France \\ ${ }^{b}$ University of Glasgow and Higher School of Economics, St Petersburg
}

\begin{abstract}
Satellites launched by independent spacefaring agencies and firms create space congestion and collision risk. Taking as benchmark the cost of a marginal reduction of the congestion rate, we discuss tax mechanisms financing a debris removal effort. We compare the non-cooperative equilibrium traffic when there is a tax on each new launch to recover cleanup costs, with the welfare optimal traffic under a centralized tax. We find that under the latter it is twice as easy to recover cleanup costs and increase traffic than under the former. We also show that a linear tax is twice as effective as a quadratic one.
\end{abstract}

Keywords: Space debris, satellites, space agents, tragedy of the commons, collision, tax system, game theory.

\section{On a tragedy of space commons}

Space is shared among all spacefaring agencies and firms, whose satellite launches create space congestion and collision risk. There is a risk that each of them launches too many satellites because they fail to internalize the impact of adding to the congestion on others than themselves. We investigate to which extent space debris mitigation can be achieved by taxing satellites launches.

Space debris refer to artificially created objects in earth orbit such as old satellites and rocket stages. It includes the fragments from their disintegration, erosion and collisions. Today there is a total of 20021 artificial objects in earth orbit including 2060 operational satellites. There are also more than 130 million debris smaller than $1 \mathrm{~cm}$, about 900000 debris 1-10 cm, and around 34000 debris larger than $10 \mathrm{~cm}$ in orbit.

\footnotetext{
${ }^{\text {in }}$ Financial support from research program In-depth UDL 2018 is gratefully acknowledged by the first author. Marc Deschamps acknowledged financial support from the Université of Franche-Comté ("Chrysalide grant"). We thank Pierre Bernhard (INRIA Sophia-Antipolis Méditerranée) for his comments, suggestions, and the more realistic model of collisions. We also wish to thank the reviewers and the editor for their detailed reading and many useful suggestions. Usual caveat apply.

${ }^{*}$ Corresponding author

Email addresses: sylvain.beal@univ-fcomte.fr (Sylvain Béal), marc.deschamps@univ-fcomte.fr (Marc Deschamps), Herve.Moulin@glasgow.ac.uk (Hervé Moulin)

$U R L:$ https://sites.google.com/site/bealpage/ (Sylvain Béal), https://sites.google.com/site/marcdeschampspage/home (Marc Deschamps), https://www.gla.ac.uk/schools/business/staff/hervemoulin/ (Hervé Moulin)
} 


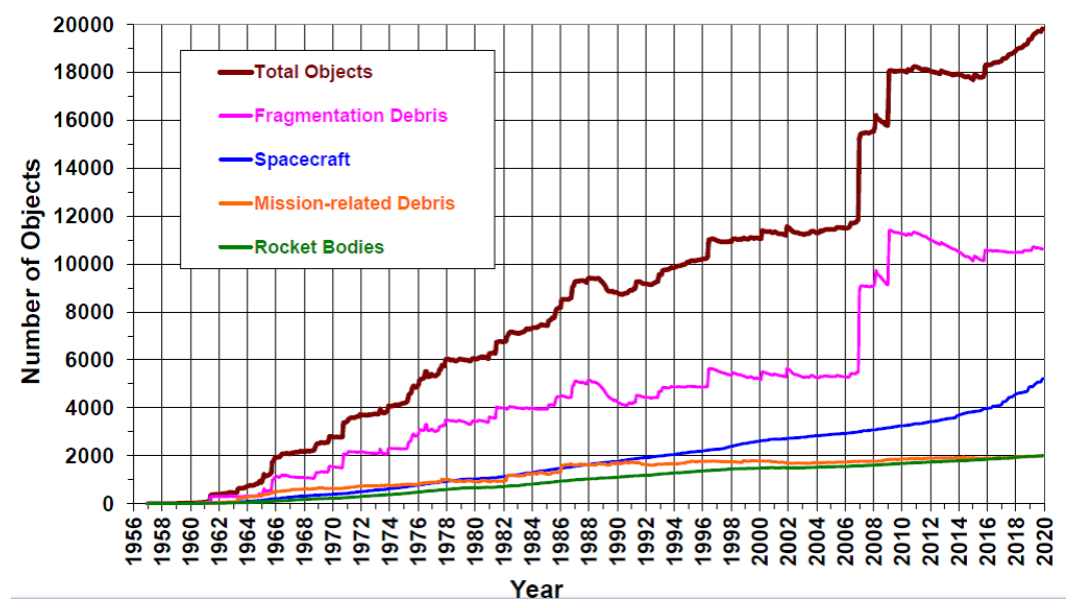

Figure 1: Evolution of the Cataloged Satellite Population (Liou, 2020)

January 2019 , number of objects and their mass (in metric tons)

\begin{tabular}{|c|c|c|c|c|c|}
\hline Selected orbits & $\begin{array}{l}\text { Fragmentation } \\
\text { debris }\end{array}$ & $\begin{array}{l}\text { Mission-related } \\
\text { debris }\end{array}$ & Rocket bodies & Payloads & $\begin{array}{l}\text { Total (including other } \\
\text { object types) }\end{array}$ \\
\hline LEO & $\begin{array}{r}8681 \\
\text { (1.5 tons) }\end{array}$ & $\begin{array}{r}769 \\
\text { (7.5 tons) }\end{array}$ & $\begin{array}{r}855 \\
(1271.7 \text { tons }) \\
\end{array}$ & $\begin{array}{r}2943 \\
(1406.5 \text { tons })\end{array}$ & $\begin{array}{r}13485 \\
\text { (2688.5 tons) }\end{array}$ \\
\hline GEO & $\begin{array}{r}3 \\
\text { (0.2 tons) }\end{array}$ & $\begin{array}{r}44 \\
\text { (1 tons) }\end{array}$ & $\begin{array}{r}65 \\
\text { (133.8 tons) }\end{array}$ & $\begin{array}{r}742 \\
\text { (646.3 tons) }\end{array}$ & $\begin{array}{r}842 \\
\text { (2 } 429.6 \text { tons) }\end{array}$ \\
\hline $\begin{array}{l}\text { Total (including objects in } \\
\text { other orbits) }\end{array}$ & $\begin{array}{r}10525 \\
(1.7 \text { tons })\end{array}$ & $\begin{array}{r}1224 \\
\text { (136.3 tons) }\end{array}$ & $\begin{array}{r}1884 \\
\text { (3258.7 tons) }\end{array}$ & $\begin{array}{r}4708 \\
\text { (5 096.1 tons) }\end{array}$ & $\begin{array}{r}22014 \\
\text { (8 } 493.8 \text { tons) }\end{array}$ \\
\hline
\end{tabular}

Note: Catalogued items come from the ESA DISCOS Database, with number of items slightly higher than those recorded by Space-track.org. Source: Adapted from ESA (2019:5]), Annual Space Environment Report: 2019.

Figure 2: Number of objects and mass (OECD, 2020)

Satellites can avoid collisions with the larger debris by slightly changing their trajectories. Their Whipple shields can also resist collisions with the smaller debris (less than $1 \mathrm{~cm}$ ). The most dangerous debris are the 1-10 cm debris which are too small and numerous to be tracked and against which Whipple shields are useless. Given the important economic impact of satellites in orbit, such collision would be disastrous. According to Adilov et al. (2018) space debris may render orbits economically unprofitable before Kessler Syndrome.

To just give a rough order of magnitude about the money involved: 1/ on LEO there is the Iridium NEXT satellite constellation which cost is estimated to 3.5 billions dollars, 2/ there is also the ISS estimated by ESA at 100 billions euros, and 3/ Wiedemann et al. (2013) estimated approximately the cost of removing 5 debris to 700 million dollars for the first year (that is before higher cost efficiency). Cleaning space is therefore a crucial issue in the astronautic community. Even, some of its members has already propose cost-benefit analysis both for space debris mitigation measures (e.g Wiedemann et al., 2004) and satellite shielding (e.g Wiedemann et al., 2008).

It should be as important for economists, but the question of cleaning space and mitigating satellites launches has received little attention in the economic literature. This assessment is shared by Weinzierl (2018), who analyzes the space economy by pointing out features that are unique to space. 
In this article, we introduce a simple model of space congestion and collision risk in which independent spacefaring agencies and firms decide on the number of satellites to launch and this interaction defines a non cooperative game, the outcome of which is a Nash equilibrium ${ }^{1}$ (simply equilibrium or non-cooperative equilibrium henceforth), i.e., a situation in which no spacefaring agency or firm can become better off by changing unilaterally its launching decision. The following two papers also approach the problem of debris removal in game theoretic terms. Klima et al. (2016) provide a very simple game-theoretical model involving only two or three space agents and few launching strategies. They focus on simulation results in an evolutionary framework where the collision risk evolves with time according to the past decisions. Singer and Musacchio (2011) analyze the cost-savings from deorbiting satellites after their mission lifetime. In their model, the costs and benefits are specified by estimations provided in the astronautic literature. Their main results are numerical simulations in which the stable coalitions of spacefaring agencies are identified, i.e. coalitions such that each coalition member is better by staying inside the coalition and each non-member is better off by remaining outside the coalition.

Our model innovates, both in terms in terms of modeling and results. We consider an arbitrary large number of spacefaring agencies and firms that decide upon launching costly satellites with the risk that some will not be operational due to collisions. Our model accounts for the launching decisions contrary to Singer and Musacchio (2011) where the decisions are limited to deorbiting efforts, and the launching possibilities are a priori not restricted contrary to Klima et al. (2016). The probability of failure of a satellite is evaluated by the probability of collision with another satellite. In order to approximate this probability, we model the space orbit as a set of boxes and apply the pigeonhole principle. A satellite is operational if there is no other satellite in its box (otherwise they destroy each other). This is a benchmark which rules out highly complex mechanics such as residual atmospheric drag, radiation pressure, periodic occultation, magnetic hysteresis, etc. A slightly more realistic approach leading to almost the same model as ours is discussed at the end of the article. Moreover, our model is flexible enough to take into account the specifications used in Singer and Musacchio (2011).

When launches are unregulated, our Lemma 1 provides a necessary and sufficient condition for the uniqueness of an equilibrium and computes the equilibrium launching decisions. It turns out that a decrease in the failure probability increases the equilibrium total number of satellites, but not necessarily for all spacefaring agencies and firms. This equilibrium total traffic is also less than in the fully regulated case, an utopian situation in which an international agency would have the power to enforce socially optimal satellite launches. In the economic field, this is a classical illustration of the tragedy of the commons in the sense of Hardin (1968). ${ }^{2}$ In order to mitigate this inefficiency, we discuss tax mechanisms financing a debris removal effort by considering as benchmark the cost of a marginal reduction of the congestion rate. We compare the non-cooperative equilibrium traffic when we tax each new launch to recover cleanup costs, with the socially optimal traffic under a

\footnotetext{
${ }^{1}$ For further details on the theory of non-cooperative games and on the concept of Nash equilibrium, we refer to Maschler et al. (2020).

${ }^{2}$ Ostrom (1990) compares theoretical predictions regarding the governance of commons with real-life outcomes. Frischmann, Marciano and Ramello (2019) provide a recent overview of the main conclusions of her framework.
} 
centralized tax. We find that under the latter it is twice as easy to recover cleanup costs and increase traffic than under the former. We also show that a linear tax is twice as effective as a quadratic one. These results are stated in Lemmas 2, 3 and 4.

Finally, our article contributes to the literature on abatement of environmental pollution, in which the stability of international agreement occupies an important place (see Carraro and Siniscalco, 1993; Barrett, 2001, for instance). And more generally to the discussion of tax policies to regulate the provision of a public bad: see for instance Hindriks and Myles (2006).

The rest of the article is organized as follows.

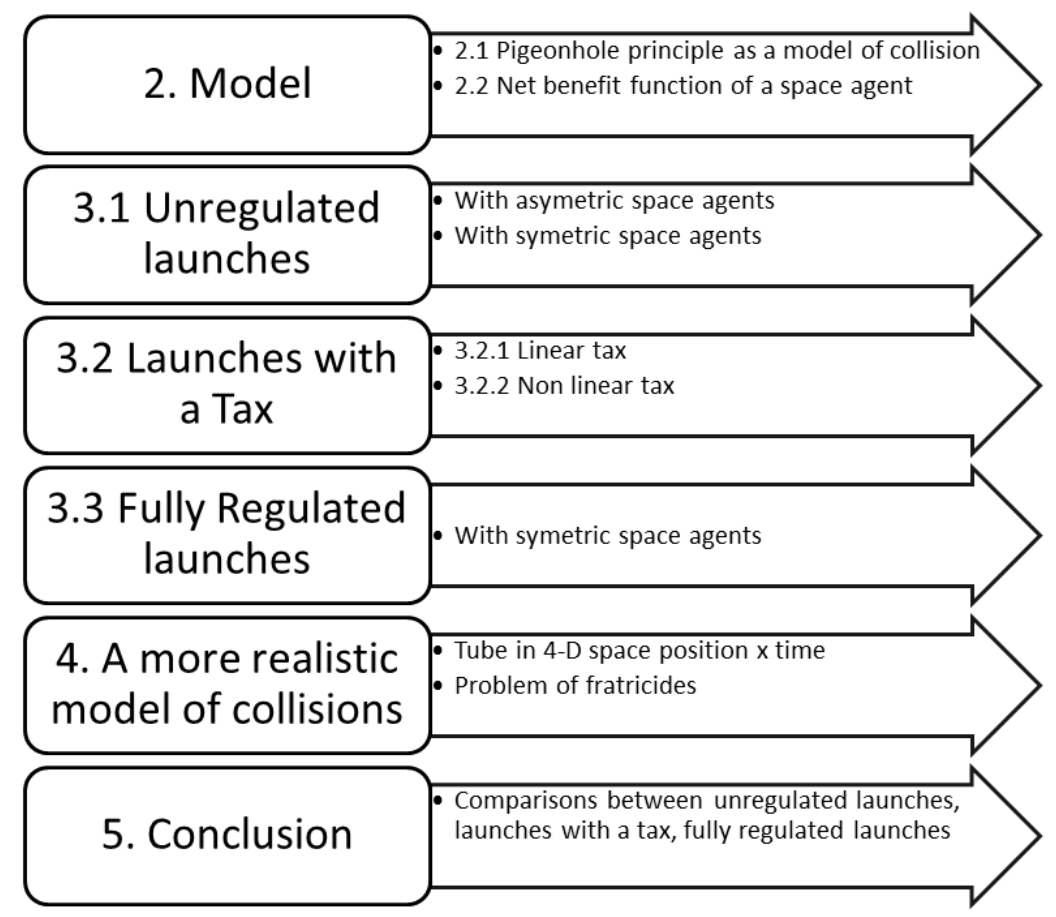

\section{Model}

This section will show the basis on which we will compare in section 3 different institutional configurations. We first propose a toy model of collision in orbital space based of the pigeonhole principle, then we define how we represent the space agents and their benefit functions.

\subsection{Collision in orbital space}

Collisions in space are modeled by a pigeonhole principle ${ }^{3}$, which provides the survival probability of a satellite or equivalently the probability that a satellite is operational. The orbit

\footnotetext{
${ }^{3}$ The pigeonhole principle is often attributed to Dirichtlet, even if it appeared two centuries before (Rittaud and Heeffer, 2014). In the conclusion, an alternative and less sketchy model is introduced.
} 
space is partitioned into $p$ boxes and the launched satellites are placed into these boxes with a uniform probability. Hence, once the first satellite is placed, the second has a probability $1 / p$ to be placed in the same box, in which case they collide and are lost. Therefore, each satellite has a probability $1-1 / p$ of surviving. The third satellite is then launched. There is again a probability $1 / p$ of being in the same box as the first satellite, independently of the previous launch. Thus the probability of survival for the first satellite becomes $(1-1 / p)^{2}$. Finally, if $x$ is the total number of satellites in orbit, then every satellite has a probability $(1-1 / p)^{x-1}$ of survival. This probability is obtained by assuming that there is no fratricide collision: it seems plausible to suppose that a satellite launcher is able to avoid to place two or more of its satellites in the same orbital box.

The survival probability can be rewritten as $(1-1 / p)^{x-1}=e^{-\tau(x-1)}$ where $\tau=-\ln (1-1 / p)$ will be interpreted as the congestion rate. This is likely to be a very small number. Basing on previous studies (e.g FP7 REVUS and $\mathrm{P}^{2}$ ROTECT) it seems reasonable to point to a failure rate of less than $10 \%$ over the operational lifetime, so we estimate that $\tau x<0.1$. Example 1 below discusses two realistic scenarios satisfying this inequality. As a consequence, we can use the approximation $e^{-\tau(x-1)} \simeq e^{\tau}(1-\tau x)$ by using the first two terms of a Taylor series. Since $\tau$ is very small, we can even use the simpler form $(1-\tau x)$ for the probability that any satellite survives. We will use it to get closed form solutions in our analysis. Thus, $\tau x^{2}$ represents the total mass of debris generated by the launch of $x$ satellites.

\subsection{Space agents}

We consider a finite set $N=\{1, \ldots, n\}$ of space agents (henceforth called agents) who are able to launch satellites. We denote by $x_{i}$ the number of satellites agent $i \in N$ launches. For analytical simplicity, and because the $x_{i}$-s are large numbers, we describe them as real numbers. We write $x=\sum_{i \in N} x_{i}$ the total number of launched satellites (the satellite total traffic).

Agent $i$ 's marginal cost of launching one more satellite is $c_{i}+\gamma_{i} x_{i}$ and its marginal benefit is normalized to 1. So, utilities are measured in "satellite equivalent"; in the economist terminology, satellite traffic is taken as the numeraire. We assume $c_{i}<1$ for otherwise agent $i$ has no interest in launching anything. The quadratic coefficient $\gamma_{i} \geq 0$ captures the decreasing returns to manage a growing fleet of satellites. It can also be interpreted as the increasing option value of funding an overseeing spatial agency, which would regulate the total satellite traffic.

If the agents choose to launch satellites $\left(x_{1}, \ldots, x_{n}\right)$, then agent $i$ 's utility (or benefit) is given by

$$
u_{i}\left(x_{1}, \ldots, x_{n}\right)=(1-\tau x) x_{i}-c_{i} x_{i}-\frac{\gamma_{i}}{2} x_{i}^{2} .
$$

In the rest of the article, without loss of generality, we shall assume that the agents are ordered by increasing order of the constant part of their marginal cost, i.e. $c_{1} \leq c_{2} \leq \cdots \leq c_{n}$.

A Nash equilibrium ${ }^{4}$ in this framework is a list $\left(x_{1}, \ldots, x_{n}\right)$ of launching decisions such that, for each agent $i \in N$ and each number of satellites $y_{i} \in \mathbb{R}_{+}$that $i$ can decide to launch, it holds

\footnotetext{
${ }^{4}$ The concept of Nash equilibrium is the most central equilibrium concept in non-cooperative game theory. In words, it is a context where no single player can obtain more payoff by changing her strategy if the others stick their own. For more details and examples we refer to Kreps (2018).
} 
that

$$
u_{i}\left(x_{1}, \ldots, x_{i-1}, x_{i}, x_{i+1}, \ldots, x_{n}\right) \geq u_{i}\left(x_{1}, \ldots, x_{i-1}, y_{i}, x_{i+1}, \ldots, x_{n}\right) .
$$

In words, if the agents decide to launch satellites according to $\left(x_{1}, \ldots, x_{n}\right)$, then agent $i$ has no incentive to change the number of satellites it launches.

\section{Three institutional configurations}

We consider three types of situations: the purely non-cooperative case where launches are not regulated, the opposite case of a central agency regulating launches, and an intermediate situation in a which a central agency sets up a tax (which can be linear or not) in order to improve upon the unregulated case.

\subsection{Unregulated launches}

The basic non-cooperative model defined by (1) is a standard tragedy of the commons problem where the failure rate is a public bad that the agents overproduce because they fail to internalize the impact of adding to the congestion on agents other than themselves.

In order to state our first result, we define the following coefficients, for each $i \in N$ :

$$
\alpha_{i}=\frac{1}{\tau+\gamma_{i}} \quad \text { and } \quad \lambda_{i}=\frac{\alpha_{i}}{\frac{1}{\tau}+\sum_{j \in N} \alpha_{j}}
$$

Lemma 1. There is a unique equilibrium $\left(x_{1}, \ldots, x_{n}\right) \in \mathbb{R}_{++}^{N}$ where all space agents are actively launching if and only if:

$$
1-c_{n} \geq \sum_{j \in N} \lambda_{j}\left(1-c_{j}\right)
$$

The equilibrium total and individual traffic levels are given by:

$$
x=\frac{1}{\tau} \sum_{j \in N} \lambda_{j}\left(1-c_{j}\right) \quad \text { and } \quad x_{i}=\alpha_{i}\left[\left(1-c_{i}\right)-\sum_{j \in N} \lambda_{j}\left(1-c_{j}\right)\right] \text { for all } i \in N .
$$

Some comments before proving Lemma 1 . The (dimensionless) number $1-c_{i}$ is a margin coefficient, the difference between the utility/value of a satellite (taken here as the numeraire) and its direct cost. Next we compute

$$
1-\sum_{j \in N} \lambda_{j}=\frac{\frac{1}{\tau}}{\frac{1}{\tau}+\sum_{j \in N} \alpha_{j}}=\frac{1}{1+\sum_{j \in N} \frac{1}{1+\frac{\gamma_{i}}{\tau}}}
$$

This difference is never smaller than $\frac{1}{n+1}$, and close to $\frac{1}{n+1}$ if the quadratic coefficients $\gamma_{i}$ are significantly smaller than $\tau$. In the latter case inequalities (2) tell us that an equilibrium with a large number of active space agents is only sustainable if their (linear) costs $c_{i}$ are close to one another. ${ }^{5}$ Similarities between marginal cost parameters is plausible at an advanced stage of the launching technology, supplied competitively by specialized firms.

\footnotetext{
${ }^{5}$ As $\gamma_{i} \ll \tau$ the $\lambda_{j}$-s are almost equal and (2) implies that the smallest margin $1-c_{i}$ (largest $c_{i}$ ) is at least $1-\frac{1}{n+1}$ times the average margin.
} 
On the contrary if the $\gamma_{i}$-s are larger than $\tau$, as in the numerical Example 1 (just after the proof below), the system (2) is compatible with fairly unequal cost parameters.

If the cost parameters are too different to have all agents active in equilibrium, the equilibrium is still unique but only a subset of agents with the lowest costs $c_{i}$ are active.

Proof. (Lemma 1) Maximizing (1), we get the first order condition $1-\tau x-\tau x_{i}-c_{i}-\gamma_{i} x_{i}=0$, or equivalently

$$
x_{i}=\frac{1-c_{i}}{\tau+\gamma_{i}}-\frac{\tau}{\tau+\gamma_{i}} x
$$

By using notation introduced before the statement of Lemma 1, the latter expression can be rewritten as

$$
x_{i}=\alpha_{i}\left(1-c_{i}\right)-\alpha_{i} \tau x .
$$

Summing (3) on $N$, we obtain

$$
x=\sum_{j \in N} \alpha_{j}\left(1-c_{j}\right)-\sum_{j \in N} \alpha_{j} \tau x
$$

and thus

$$
x=\frac{1}{\tau} \sum_{j \in N} \lambda_{j}\left(1-c_{j}\right)
$$

as desired. Replacing $x$ in (3), we get the unique equilibrium quantity

$$
x_{i}=\alpha_{i}\left[\left(1-c_{i}\right)-\sum_{j \in N} \lambda_{j}\left(1-c_{j}\right)\right]
$$

for each $i \in N$. It remains to ensure that all agents are active at equilibrium, which amounts to have $x_{i}>0$ for each $i \in N$ in (4), and leads immediately to

$$
1-c_{i} \geq \sum_{j \in N} \lambda_{j}\left(1-c_{j}\right)
$$

for each $i \in N$. Since $c_{1} \leq c_{2} \leq \cdots \leq c_{n}$, we get condition (2).

Let us illustrate the mechanisms behind Lemma 1 by the following example.

Example 1. In this academic example, to keep things simple, we only limit ourselves to five space agents, even if the example can be extended easily to more agents as underlined at the end of the example. So let us set $N=\{1, \ldots, 5\}$. For each $i \in\{1,2,3,4,5\}$, the cost parameters are given by

$$
c_{i}=\left(\frac{i}{6}\right)^{2}+\frac{3}{10} \quad \text { and } \quad \gamma_{i}=5 i \times 10^{-4} \text {. }
$$

These values are essentially chosen so that the space agents differ in the efficiency of their launching technologies (since both $c_{i}$ and $\gamma_{i}$ depend on $i$ ). Agent 1 owns the most efficient technology while agent 5 owns the least efficient technology. Furthermore, for each space agent $i$, note that the 
quadratic coefficient $\gamma_{i}$ is small and much less than the constant part of the marginal cost $c_{i}$. This implies that the extra cost of launching one more satellite increases only very little with the satellite fleet. In the remainder, we are considering three scenarios described by three congestion rate values for $\tau$.

In the first scenario, suppose that $\tau=2 \times 10^{-5}$, i.e. a moderate congestion rate. In this situation, condition (2) is satisfied for all agents except for the agent 5 which has the least efficient launching technology. Therefore agent 5 will launch $x_{5}=0$ satellite and everything happens as if agents 1,2,3 and 4 are involved in a four-agent game. In this game, condition (2) is still satisfied for all of them, meaning that they are all active in the equilibrium of the five-agent game in which agent 5 is inactive. More specifically, from (4), we get the following equilibrium quantities: $x_{1}=1211.2$, $x_{2}=535.8, x_{3}=268.1$ and $x_{4}=105.5$. The resulting total satellite fleet $x=2120.6$ is close to the current total number of satellites. The probability of losing a satellite is then $\tau x=4.04 \%$.

In the second scenario, suppose that space congestion becomes twice as high: $\tau=4 \times 10^{-5}$. At equilibrium, only the first four agents are active once again, with the following equilibrium quantities: $x_{1}=1055, x_{2}=473.3, x_{3}=231$ and $x_{4}=79.8$. The total satellite fleet $x=1839.1$ is reduced by $13 \%$ but is not far from real current level. Not surprisingly, the probability of losing a satellite more than doubles to $9.2 \%$.

In the third scenario, we would like to highlight how the congestion rate can influence the number of active space agents at equilibrium. In order to do so, consider a considerable, but less plausible, increase of the space congestion: $\tau=4 \times 10^{-4}$. In this case, condition (2) is not satisfied for agents 4 and 5. Space congestion is now high enough to drive out of the space activities the agent with the second worst launching technology. In the four-agent game obtained after removing agent 5, condition (2) remains unsatisfied for agent 4, while in the three-agent game obtained after the further removal of agent 4, condition (2) is satisfied for agents 1, 2 and 3. As a consequence, coming back to the initial five-agent game, both agents 4 and 5 will be inactive at equilibrium. The resulting equilibrium quantities are: $x_{1}=425.3, x_{2}=213.9, x_{3}=84.5, x_{4}=0, x_{5}=0$. This yields a decrease of the total fleet and an increase in the failure probability, both substantial $(x=723.7$ and $\tau x=28.9 \%$ ).

Finally, this example can account, somehow artificially, for more space agents by adding agents whose technologies are even less effective than agent 5's technology. In this case, these new agents will be inactive at equilibrium in each of our three scenarios and would not influence the aforementioned equilibrium quantities.

The equilibrium quantities have a simpler form in the following two special cases.

Corollary 1. If $c_{i}=\bar{c}$ for all $i \in N$, all agents are active in equilibrium and

$$
x=\frac{1-\bar{c}}{\tau+\frac{1}{n} H(\tau+\gamma)} \quad \text { and } \quad x_{i}=\frac{H(\tau+\gamma)}{\tau+\gamma_{i}} \frac{1}{n} x
$$

where $H(\tau+\gamma)$ is the harmonic mean of the $n$ coefficients $\left(\tau+\gamma_{i}\right), i \in N$.

Corollary 2. If $\gamma_{i}=\bar{\gamma}$ for all $i \in N$, the equilibrium has everyone active if and only if

$$
\frac{1-c_{i}}{1-\bar{c}} \geq \frac{1}{1+\frac{1}{n}\left(1+\frac{\bar{\gamma}}{\tau}\right)}
$$


and in this case it is

$$
x=\frac{1-\bar{c}}{\tau+\frac{1}{n}(\tau+\bar{\gamma})} \quad \text { and } \quad x_{i}=\frac{1}{\tau+\bar{\gamma}}\left(1-c_{i}-\tau x\right)
$$

Comparing (6) and (8), and remembering that the harmonic mean is below the arithmetic one, we see that, starting from the fully symmetric case where $c_{i}$ and $\gamma_{i}$ are constant, a mean preserving spread of the $\gamma_{i}$-S will increase total traffic. A mean preserving spread of the $c_{i}$-s will not change total traffic as long as the $c_{i}$-s remain close enough, but it will quickly shut out some agents hence reducing total traffic.

Comparative statics. Decreasing the probability of failure $\tau$ increases total equilibrium traffic in the general case, but not necessarily for each agent. This is true for all agents if the $\gamma_{i}$-s are constant, but not necessarily if only the $c_{i}$-s are constant.

Decreasing $c_{i}$ for some agent $i$ also increases total traffic in the general case, but not necessarily for each agent. However the latter is true if either the $c_{i}$-s or the $\gamma_{i}$-s are constant.

The effect of decreasing $\gamma_{i}$ for some $i$ on total and individual traffic is ambiguous in the general case, not so if the $c_{i}$-s are constant.

Finally if we add one agent, the effect is also ambiguous on total equilibrium traffic, even in the fully symmetric case, because the new agent can break that symmetry. Of course the ambiguity disappears if the new agent has the average parameters.

\subsection{Paying for cleanup: linear and non linear tax}

In this section, we ask if a feasible cleanup financed by the agents themselves would actually allow total equilibrium traffic to increase. If the cleanup is too expensive, that may not be the case. Our simple model yields a precise statement. We start from a fully symmetric problem where $c_{i}=\bar{c}$ and $\gamma_{i}=\bar{\gamma}$ for all $i \in N$. Hence, the total equilibrium traffic is given by (8). The overseeing authority (thereafter the Agency) is considering a reduction of the failure probability/congestion parameter from $\tau$ to $\tau-\Delta \tau$, which can be achieved at a cost $\Delta C .{ }^{6}$

\subsubsection{Linear tax}

Suppose the Agency sets a linear $\operatorname{tax} \theta$ on each satellite launched, so as to recover $\Delta C$. We study the impact on total satellite traffic in the resulting new equilibrium of the game. So let $x$ be the initial equilibrium traffic given by (8). When the tax is added, parameter $\bar{c}$ raises to $\bar{c}+\theta$, and the new total traffic is

$$
x^{\Delta}=\frac{1-\bar{c}-\theta}{\left(1+\frac{1}{n}\right)(\tau-\Delta \tau)+\frac{1}{n} \bar{\gamma}},
$$

and the Agency collects $\theta x^{\Delta}$ from the tax. Now, we have to compute $\theta$ so that $x^{\Delta}=x$, i.e. in order to keep constant the equilibrium satellite traffic. We get

$$
x^{\Delta}=x \Longleftrightarrow \frac{\theta}{1-\bar{c}}=\frac{\left(1+\frac{1}{n}\right) \Delta \tau}{\left(1+\frac{1}{n}\right) \tau+\frac{1}{n} \bar{\gamma}} \Longleftrightarrow \theta=\left(1+\frac{1}{n}\right) x \Delta \tau .
$$

\footnotetext{
${ }^{6}$ We do not specify/discuss the technology which enables such a change.
} 
From that $\operatorname{tax} \theta$ the Agency collects

$$
\theta x=\left(1+\frac{1}{n}\right) x^{2} \Delta \tau
$$

Therefore, if $\Delta C<\left(1+\frac{1}{n}\right) x^{2} \Delta \tau$, it is possible for the Agency to choose a tax in the interval $\left[\Delta C / x,\left(1+\frac{1}{n}\right) x \Delta \tau\right)$ so as to generate a higher traffic than $x$ and to recoup the cleanup cost. We summarize these steps and remarks in the following Lemma.

Lemma 2. A flat tax is enough to recover cleanup costs and increase traffic if and only if

$$
\frac{\Delta C}{\Delta \tau} \leq \frac{n+1}{n} x^{2}
$$

\subsubsection{Non linear tax}

Assume now the Agency puts pressure on the quadratic part of the costs: agent $i$ is charged $(1 / 2) \delta x_{i}^{2}$, so that $\bar{\gamma}$ raises to $\bar{\gamma}+\delta$. The tax is thus progressive: the per-satellite tax is higher for space agents launching more satellites. We assume as above a reduction $\Delta \tau$ of the congestion parameter, and compute similarly the tax rate ensuring that the traffic remains constant:

$$
x^{\Delta}=\frac{1-\bar{c}}{\left(1+\frac{1}{n}\right)(\tau-\Delta \tau)+\frac{1}{n}(\bar{\gamma}+\delta)} .
$$

It is easy to find that $x^{\Delta}=x$ if and only if $\delta=(n+1) \Delta \tau$. Then the Agency collects

$$
\frac{1}{2} \delta \sum x_{i}^{2}=\frac{1}{2 n} \delta x^{2} \text {. }
$$

This yields the following Lemma.

Lemma 3. A quadratic tax is enough to recover cleanup costs and increase traffic if and only if

$$
\frac{\Delta C}{\Delta \tau} \leq \frac{n+1}{2 n} x^{2}
$$

We can compare Lemmas 2 and 3. The progressive tax is more demanding than the flat tax in terms of technology, as measured by the ratio $T=\Delta \tau / \Delta C$ required. This ratio can be seen as the efficiency of technology, which reply to the question: how much is it possible to reduce congestion for a given price? (i.e $\Delta \tau=T . \Delta C$ ). These results show that a progressive tax requires a technology twice as efficient. It is also less efficient in the sense that, for a fixed technological level $T$, a traffic preserving financial equilibrium requires a population satellite $x=\sqrt{2 n / T(n+1)}$, $41 \%$ larger with the progressive tax as compared to the flat tax. Indeed, from Lemma 2 we can find $x^{2}=\frac{n}{T} \cdot(n+1)$ whereas we have $x^{2}=\frac{2 n}{T}$. $(n+1)$ from Lemma 3 : a difference of $\sqrt{2}$ between the two.

The example below illustrates the thresholds in Lemmas 2 and 3.

Example 2. Consider the first scenario in example 1, where $\tau=2 \times 10^{-5}$. For the sake of comparison with the results in this section, we consider five symmetric space agents such that $c_{i}=\bar{c}$ and $\gamma_{i}=\bar{\gamma}$ for each $i \in\{1, \ldots, 5\}$. From (5), this means that each space agent $i$ is such that

$$
c_{i}=\bar{c}=0.606 \quad \text { and } \quad \gamma_{i}=\bar{\gamma}=0.0015 .
$$


The resulting equilibrium traffic is $x=1217.42$, with each agent launching quantity 243.48 , and the failure probability is $\tau x=2.43 \%$. In the rest of this example, imagine that the target is to reduce the failure probability from $2.43 \%$ to $1.43 \%$, which corresponds to a decrease by $41.1 \%$ of the congestion parameter $\tau$. Furthermore, assume that the cost $\Delta C$ necessary to attain this objective is exactly the cost that preserves the total traffic.

According to Lemma $2, \Delta C=14.62$. Such a cost represents only $0.66 \%$ of the total cost generated by launching $x$. In this case, the flat tax is $\theta=0.012$, which represents only an increase by $2 \%$ of the marginal cost $\bar{c}$ in (10).

According to Lemma $3, \Delta C=7.31$ and the quadratic tax is $\theta=0.006$, which represents an increase by $400 \%$ of the quadratic parameter $\bar{\gamma}$ in (10).

\subsection{Fully regulated launches}

In this section, we assume that the Agency can centrally regulate launching, and that its mission is to maximize total welfare, still measured in the satellite numeraire. We can develop similar computations allowing heterogeneous parameters $c_{i}$ and $\gamma_{i}$, but for brevity we concentrate on the fully symmetric case. The total sum of utilities is

$$
\sum_{i \in N} u_{i}\left(x_{1}, \ldots, x_{n}\right)=(1-\tau x) x-\sum_{i \in N}\left(c_{i} x_{i}+\frac{1}{2} \gamma_{i} x_{i}^{2}\right)=(1-\tau x) x-\left(\bar{c} x+\frac{1}{2 n} \bar{\gamma} x^{2}\right) .
$$

The first order condition $(1-2 \tau x)-\bar{c}-\bar{\gamma} x / n=0$ can be rearranged to obtain the optimal centralized traffic $x^{*}$ :

$$
x^{*}=\frac{1-\bar{c}}{2 \tau+\frac{1}{n} \bar{\gamma}} .
$$

This total traffic is less than the total traffic (8) in the unregulated case, but it is significantly below only if $n \tau$ is commensurate to $\bar{\gamma}$ since

$$
\frac{x^{\text {dect }}-x^{*}}{x^{\text {dect }}}=\frac{\frac{n-1}{n}}{2+\frac{\bar{\gamma}}{n \tau}} \simeq \frac{1}{2+\frac{\bar{\gamma}}{n \tau}},
$$

where $x^{\text {dect }}$ stands for the equilibrium traffic given by (8). As in section 3.2 , we can also compute the cost effectiveness of financing cleanup by a flat $\operatorname{tax} \theta$ or a quadratic $\operatorname{tax} \delta$. With a flat tax, the analog of equation (9) gives $\theta=2 x \Delta \tau$ and the Agency collects $2 x^{2} \Delta \tau$. With a progressive tax, we get similarly $\delta=2 n \Delta \tau$ and a collection of $x^{2} \Delta \tau$. This entails the next Lemma.

Lemma 4. Under centralized launching:

(i) A flat tax is enough to recover cleanup costs and increase traffic if and only if

$$
\frac{\Delta C}{\Delta \tau} \leq 2 x^{2}
$$

(ii) A quadratic tax is enough to recover cleanup costs and increase traffic if and only if

$$
\frac{\Delta C}{\Delta \tau} \leq x^{2}
$$


When Lemma 4 is compared to Lemmas 2 and 3, a possible interpretation is that in the unregulated regime, the critical threshold of technology level for a cleanup technology financed by either a flat or a quadratic cost, is approximately twice higher as in the centralized regulated regime provided that $n$ is not too small.

The example below illustrates Lemma 4 as in example 2 .

Example 3. As in example 2, assume that $\tau=2 \times 10^{-5}$ and that the technology of each agent $i \in\{1, \ldots, 5\}$ is described by (10). In the fully regulated case, the equilibrium traffic is then $x=1160.13$, which represents a decrease by $4.71 \%$ compared to the situation of unregulated launches. The corresponding failure probability is $\tau x=2.32 \%$. As in example 2 , imagine that the target is to reduce the failure probability by one point, from $2.32 \%$ to $1.32 \%$, which corresponds to a decrease by $43.1 \%$ of $\tau$. Once again, we assume that the cost $\Delta C$ necessary to attain this objective is exactly the cost that preserves the total traffic.

According to Lemma $4, \Delta C=23.2$ if a flat tax is used. The flat tax is then $\theta=0.02$, which represents only an increase by $3.3 \%$ of the marginal cost $\bar{c}$ in (10). Similarly, $\Delta C \leq 11.6$ is a quadratic tax is used. In this case, the quadratic tax is $\theta=0.01$, which represents an increase by $667 \%$ of the quadratic parameter $\bar{\gamma}$ in (10).

This section proved, in our model, the usual conclusions in economics. If space agents are totally free to do what they want, they do not consider the bads they create (i.e debris): it is the unregulated regime. As economists we are used to study the centralized case first in order to achieve two goals. The first one is to estimate the bad created in the unregulated case. The second one is to determine a desirable tax level when agents are free to choose how many launches they want but have to pay a tax for each launch. The main result in this section is that a flat tax is better than a progressive tax in our model.

\section{A more realistic model of collisions}

In this section, we discuss a variant of the model of collisions developed in section 2.1. As in our model, we keep the assumption that all satellites are identical. Each occupies a "tube" in 4-D space position $\times$ time. Suppose that the volume of one such tube is $v$. Collisions between satellites are intersections of two of these 4-D tubes. The probability for a given satellite of colliding with any of $z$ satellites is evaluated by a function (not specified at this point) of the ratio of the total volume $z v$ of their tubes to the total available volume $V$ of the portion of 4-D space where all live. Let $\varepsilon=\frac{v}{V}$. In this variant, we can account for fratricides that follow with the same law as other collisions. Finally, it is useful to introduce a parameter $x_{0}$, which represents the number of objects (satellites and debris) already in orbit at the start of the analysis. Now, the probability $P$ of a satellite colliding with any of $z$ other objects during its life time will therefore be taken as a function $P=f(\varepsilon z)$ with $f(0)=0$, and $\varepsilon$ a very small number, so that a reasonable approximation is given by a first order expansion, where $\tau=f^{\prime}(0) \varepsilon$ is a small positive number: $P \simeq \tau z$. These 
will be considered independent events. Thus, launching $x_{i}$ satellites results in an expected number of "operational" satellites given by

$$
\left[1-f\left(\varepsilon\left(x_{0}+\sum_{j \in N \backslash\{i\}} x_{j}\right)\right)-2 f\left(\varepsilon x_{i}\right)\right] x_{i}=\left[1-\tau\left(x_{0}+x+x_{i}\right)\right] x_{i}
$$

if fratricides happen with the same probability as other collisions, and

$$
\left[1-f\left(\varepsilon\left(x_{0}+\sum_{j \in N \backslash\{i\}} x_{j}\right)\right)\right] x_{i}=\left[1-\tau\left(x_{0}+x-x_{i}\right)\right] x_{i}
$$

if fratricides are ruled out.

Introduce the fratricide index $\phi=1$ if fratricides are present and $\phi=-1$ if they are ruled out, and the coefficients

$$
a_{i}=c_{i}+\tau x_{0} \quad \text { and } \quad b_{i}=\gamma_{i}+2 \phi \tau .
$$

Coupled with the cost function $c_{i} x_{i}+\gamma_{i} x_{i}^{2} / 2$, this leads to a utility of the form

$$
u_{i}\left(x_{1}, \ldots, x_{n}\right)=(1-\tau x) x_{i}-a_{i} x_{i}-\frac{1}{2} b_{i} x_{i}^{2} .
$$

The optimal quantities in the unregulated (Lemma 1) and fully regulated situations remain the same in this variant of our model, provided that $a_{i}$ and $b_{i}$ replace $c_{i}$ and $\gamma_{i}$ for all agent $i \in N$. Nonetheless, the comparative static would be affected affected since parameter $\tau$ enters into $a_{i}$ and $b_{i}$.

In that model, the cleaning effect may be taken as lowering $x_{0}$. Calculations very similar to those performed in the this article show that the technology level $T=\Delta x_{0} / \Delta C$ required to preserve total traffic with a progressive tax is $2 / \tau x$, again twice that required with the flat tax.

The aim of this section was only to underline that even if our model of collision in orbital space of section 2 is a toy model, we can find some arguments to consider it as a non totally physically unreal model. This model already presents important issues in various situations that make sense from an economical perspective. We think this is a preliminary step before scientists agree on the collision model to use.

\section{Conclusion}

Nobody is able today to do without the services of the satellites. As an example, A. Wagner (Airbus Defence and Space) recently reported to J-L. Fugit, a French deputy responsible for a parliamentary note on satellites and their applications, that every smartphone user daily uses the services provided by 40 satellites. Another example, given by Ch. Bonnal (CNES), concern the global warming and underlines that on the 50 parameters to analyze it, 29 are only accessible from space. This dependence on satellites is a major feature of our modern economies and it is growing every day, given the proliferation of applications requiring satellites and the lower cost of access to space. 
However, if nothing is done, the space debris problem (which currently leads the main satellite operators to conduct avoidance maneuvers on average once a week) may become an economic emergency for both space operators and all downstream markets that depend on them.

As economists, we consider that our discipline can help to protect the global common good of space while preserving the dynamics of the "New Space". The tragedy announced is not inevitable if we agree on the institutional measures that can prevent it. By using our numeric example in examples 2 and 3, our conclusions can be illustrated by a table:

\begin{tabular}{|l|c|c|}
\cline { 2 - 3 } \multicolumn{1}{c|}{} & Unregulated launches & Fully regulated launches \\
\hline Total traffic & 1217.42 & 1160.13 \\
\hline Failure Probability (FP) & $2.42 \%$ & $2.32 \%$ \\
\hline $\begin{array}{l}\text { Linear tax for a 1pt FP decrease without } \\
\text { reducing traffic }\end{array}$ & $+2 \%$ of $\bar{c}$ & $+3.3 \%$ of $\bar{c}$ \\
\hline $\begin{array}{l}\text { Quadratic tax for a 1pt FP decrease with- } \\
\text { out reducing traffic }\end{array}$ & $+400 \%$ of $\bar{\gamma}$ & $+667 \%$ of $\bar{\gamma}$ \\
\hline
\end{tabular}

This problem is currently taking a turn since we are seeing the sending of many cubsats (around 500 per year) and the arrival of mega-constellations of satellites in Low Earth Orbit, such as SpaceX, Amazon or OneWeb who respectively wish send 12000 satellites, 3000 satellites and 1000 satellites.

This change of magnitude of the problem will inevitably change the rules governing space and as economists we wish to participate in the important reflection on these. We actually work on mechanisms which provide the compatibility of the individual incentives of agents who launch satellites and the common good that represents a safe space available for all. We began to examine this topic with some members of INRIA Sophia Antipolis and CNES, but we naturally wish to engage discussions and work with all bodies that send satellites or plan to offer ways to remove debris. Our approach seems to us complementary to those followed by astronautic community (e.g Neish and Goka , 2001; Wiedemann et al., 2013).

Adilov, N., Alexander, P., Cunningham, B., 2018. An economic "Kessler Syndrome": A dynamic model of earth orbit debris. Economics Letters 166, 79-82.

Barrett, S., 2001. International cooperation for sale. European Economic Review 45, 1835-1850.

Carraro, C., Siniscalco, D., 1993. Strategies for the international protection of the environment. Journal of Political Economy 52, 309-328.

Frischmann, B., Marciano, A., Ramello Giovanni Battista 2019. Tragedy of the Commons after 50 Years. Journal of Economic Perspectives, 33(4), 211-228.

Hardin, G., 1968. The tragedy of the commons. Science 162, 1243-1248.

Hindriks, J., Myles, G. D., 2006. Intermediate Public Economics. MIT Press.

Klima, R., Bloembergen, D., Savani, R., Tuyls, K., Hennes, D., Izzo, D., 2016. Space debris removal: A game theoretic analysis. Games 7.

Kreps, D., 2018. Nash Equilibrium. The New Palgrave Dictionary of Economics, Macmillan, 9251-9258.

Liou, J-C., 2020. The 2019 U.S Government Orbital Debris Mitigation Standard Practices. 57th Session of the Scientific and Technical Subcommittee. Committee on the Peaceful Uses of Outer Space, United Nations, 3-14 February, Vienna.

Maschler, M., Solan, E., Zamir, S., 2020. Game Theory. Cambridge University Press, 2nd edition. 
Neish, M., Goka, T., 2001. Costs and benefits of space debris mitigation. Proceedings of the Third European Conference on Space Debris, 19 - 21 March 2001, Darmstadt, Germany. Ed.: Huguette Sawaya-Lacoste. ESA SP-473, Vol. 2, Noordwijk, Netherlands: ESA Publications Division, 745-750

OECD, 2020. Space Sustainability. The Economics of Space Debris in Perspectie. OECD Science, Technology and Industry. Policy Papers, April, $\mathrm{n}^{\circ} 87$

Ostrom, E., 1990. Governing the Commons: The Evolution of Institutions for Collective Actions. Cambridge University Press.

Rittaud, B., Heeffer, A., 2014. The pigeonhole principle, two centuries before Dirichlet. The Mathematical Intelligencer 36, 27-29.

Singer, M. J., Musacchio, J. T., 2011. An international environmental agreement for space debris mitigation among asymmetric nations. Acta Astronautica 68, 326-337.

Wiedemann, C., Oswald, M., Bendisch, J., Sdunnus, H., Vörsmann, P., 2004. Cost and benefit analysis of space debris mitigation measures. Acta Astronautica 55, 311-324.

Wiedemann, C., Oswald, M., Stabroth, S., Alwes, D., Vörsmann, P., 2008. Cost and benefit analysis of satellite shielding. Acta Astronautica 63, 136-145.

Wiedemann, C., Flegel, S., Möckel, M., Gelhaus, J., Braun, V., Kebschull, c., Kreiseil, J., Metz, M., Vörsmann, P., 2013. The economics of the control of the space debris environment. Proc. 6th European Conference on Space Debris, Darmstadt, paper 2818614, April, ESA-SP-723, August 2013.

Weinzierl, M., 2018. Space, the final economic frontier. Journal of Economic Perspectives 32, 173-192. 